Article

\title{
Simultaneous and Non-Simultaneous Quenching for a System of Multi-Dimensional Semi-Linear Heat Equations
}

\author{
Ratinan Boonklurb ${ }^{1, * \mathbb{C}}$, Tawikan Treeyaprasert ${ }^{2}$ and Aong-art Wanna ${ }^{1}$ \\ 1 Department of Mathematics and Computer Science, Faculty of Science, Chulalongkorn University, \\ Bangkok 10330, Thailand; 6172090023@student.chula.ac.th \\ 2 Department of Mathematics and Statistics, Faculty of Science and Technology, \\ Thammasat University Rangsit Center Khlong Luang, Pathum Thani 12120, Thailand; tawikan@tu.ac.th \\ * Correspondence: ratinan.b@chula.ac.th
}

Received: 20 November 2020; Accepted: 14 December 2020; Published: 14 December 2020

\begin{abstract}
This article deals with finite-time quenching for the system of coupled semi-linear heat equations $u_{t}=u_{x x}+f(v)$ and $v_{t}=v_{x x}+g(u)$, for $(x, t) \in(0,1) \times(0, T)$, where $f$ and $g$ are given functions. The system has the homogeneous Neumann boundary conditions and the bounded nonnegative initial conditions that are compatible with the boundary conditions. The existence result is established by using the method of upper and lower solutions. We obtain sufficient conditions for finite time quenching of solutions. The quenching set is also provided. From the quenching set, it implies that the quenching solution has asymmetric profile. We prove the blow-up of time-derivatives when quenching occurs. We also find the criteria to identify simultaneous and non-simultaneous quenching of solutions. For non-simultaneous quenching, the corresponding quenching rate of solutions is given.
\end{abstract}

Keywords: simultaneous quenching; nonsimultaneous quenching; semi-linear parabolic system

MSC: 35K05; 35K51; 35K55; 35K58; 35K67

\section{Introduction}

Since the study of quenching phenomena for the parabolic equations was begun in 1975 by Kawarada [1], a lot of works along this direction, its generalization and its variation have been contributed. For example, in 2002, De Pablo et al. [2] studied the coupled parabolic system

$$
\left.\begin{array}{l}
u_{t}=u_{x x}-v^{-p}, \quad(x, t) \in(0,1) \times(0, T) \\
v_{t}=v_{x x}-u^{-q}, \quad(x, t) \in(0,1) \times(0, T)
\end{array}\right\}
$$

subject to the Neumann boundary conditions and initial conditions

$$
\begin{aligned}
& u_{x}(0, t)=u_{x}(1, t)=v_{x}(0, t)=v_{x}(1, t)=0, \quad t \in(0, T), \\
& u(x, 0)=u_{0}(x)>0 \text { and } v(x, 0)=v_{0}(x)>0, \quad x \in[0,1] .
\end{aligned}
$$

In Reference [2], the solution $u$ or $v$ of Equation (1) is said to quench in a finite time if there exists $T_{q}<\infty$ such that $u$ or $v$ exists in the classical sense and is positive for all $0 \leq t<T_{q}$, while

$$
\liminf _{t \rightarrow T_{q}} \min _{0 \leq x \leq 1}\{u(x, t)\}=0 \text { or } \liminf _{t \rightarrow T_{q}} \min _{0 \leq x \leq 1}\{v(x, t)\}=0 .
$$


The System (1) is said to quench simultaneously if both $u$ and $v$ quench at the same finite time $T_{q}$. However, if only one of the solutions quenches in a finite time $T_{q}$, then it is called non-simultaneous quenching. The main results of [2] can be summarized as follows.

- If $p, q \geq 1$, then any quenching solutions of (1) is simultaneous; if $p \leq 1 \leq q$, then any quenching solutions of (1) is non-simultaneous with $u$ being strictly positive; if $p, q \leq 1$, then there exists $\left(u_{0}, v_{0}\right)$ such that simultaneous quenching occurs.

- If quenching is non-simultaneous, and, for instance, $v$ is the unique quenching component, then $v(0, t) \sim\left(T_{q}-t\right)$. Otherwise:

(a) $u(0, t) \sim\left(T_{q}-t\right)^{\frac{p-1}{p q-1}}, v(0, t) \sim\left(T_{q}-t\right)^{\frac{q-1}{p q-1}}$ if $p, q>1$ or $p, q<1$;

(b) $u(0, t), v(0, t) \sim\left(T_{q}-t\right)^{\frac{1}{2}}$ if $p=q=1$;

(c) $u(0, t) \sim\left(T_{q}-t\right)\left|\log \left(T_{q}-t\right)\right|^{\frac{p}{p-1}}, v(0, t) \sim\left|\log \left(T_{q}-t\right)\right|^{\frac{-1}{p-1}}$ if $p>q=1$.

Note that $f \sim g$ means there exist finite positive constants $c_{1}$ and $c_{2}$ such that $c_{1} g(x) \leq f(x) \leq c_{2} g(x)$, for all $x \in D_{f} \cap D_{g}$. Furthermore, the blow-up of $u_{t}$ and $v_{t}$ at the quenching time were also proved. Since they can prove that $u_{t}$ and $v_{t}$ are decreasing functions of $t$, the blow-up of them means $u_{t} \rightarrow-\infty$ and $v_{t} \rightarrow-\infty$ as $t$ approaches the quenching time.

In 2016, Pei and Li [3] worked on the coupled parabolic system

$$
\left.\begin{array}{ll}
u_{t}(x, t)=\Delta u-f(v(x, t)), & (x, t) \in \Omega \times(0, T), \\
v_{t}(x, t)=\Delta v-f(u(x, t)), & (x, t) \in \Omega \times(0, T),
\end{array}\right\},
$$

where $T>0$ and $\Omega \subset \mathbb{R}^{n}$ is a bounded domain with smooth boundary subject to the Dirichlet boundary conditions and initial conditions

$$
u=v=1,(x, t) \in \partial \Omega \times(0, T) \text { and } u(x, 0)=u_{0}(x), v(x, 0)=v_{0}(x), x \in \bar{\Omega} .
$$

They derived the quenching rate in the case of non-simultaneous quenching.

Later, in 2019, Chan [4] studied the semi-linear parabolic system

$$
\left.\begin{array}{ll}
u_{t}-\Delta u=\frac{a}{1-v(0,0, t)}, & \text { in } D \times(0, \Gamma), \\
v_{t}-\Delta v=\frac{b}{1-u(0,0, t)}, & \text { in } D \times(0, \Gamma),
\end{array}\right\}
$$

subject to the Dirichlet boundary conditions and initial conditions

$$
u(x, y, t)=v(x, y, t)=0, \text { on } \partial D \times(0, \Gamma) \text { and } u(x, y, 0)=v(x, y, 0)=0, \text { on } \bar{D},
$$

where $D=(-1,1) \times(-1,1), \Gamma \in(0, \infty]$, and $a, b$ are positive constants such that $a \leq b$. The definition of quenching was different from References [2,3]. In Reference [4], the solution $u$ or $v$ is said to quench if there exists a finite time $\Gamma$ such that

$$
\max _{(x, y) \in \bar{D}}\{u(x, y, t)\} \rightarrow 1^{-} \text {or } \max _{(x, y) \in \bar{D}}\{v(x, y, t)\} \rightarrow 1^{-} \text {as } t \rightarrow \Gamma^{-} .
$$

He proved that the solutions quench simultaneously and approximated critical values $a^{*}$ and $b^{*}$. This $a^{*}$ and $b^{*}$ associate with the existence of the solutions of their steady state system.

Let $T>0$. In this article, we consider the system of coupled semi-linear heat equations

$$
\left.\begin{array}{l}
u_{t}(x, t)=u_{x x}(x, t)+f(v(x, t)),(x, t) \in(0,1) \times(0, T), \\
v_{t}(x, t)=v_{x x}(x, t)+g(u(x, t)),(x, t) \in(0,1) \times(0, T),
\end{array}\right\},
$$


subject to the Neumann boundary conditions

$$
u_{x}(0, t)=u_{x}(1, t)=0 \text { and } v_{x}(0, t)=v_{x}(1, t)=0, t \in(0, T]
$$

and the initial conditions

$$
u(x, 0)=u_{0}(x) \text { and } v(x, 0)=v_{0}(x), x \in[0,1] .
$$

We assume that the initial conditions are nonnegative, bounded, smooth, and compatible with the boundary conditions. Let $c$ be a positive constant. The given functions $f$ and $g$ are positive and satisfy the following conditions:

Hypothesis 1. $f(s), g(s) \in C^{2}([0, c))$;

Hypothesis 2. $\lim _{s \rightarrow c^{-}} f(s)=\infty$ and $\lim _{s \rightarrow c^{-}} g(s)=\infty$;

Hypothesis 3. $f^{\prime}(s)>0$ and $g^{\prime}(s)>0$ for $s \in[0, c)$;

Hypothesis 4. $f^{\prime \prime}(s) \geq 0$ and $g^{\prime \prime}(s) \geq 0$ for $s \in[0, c)$.

Throughout this work, we assume that $u_{0}(x)<c, v_{0}(x)<c$, and they satisfy

$$
\begin{aligned}
u_{0}^{\prime \prime}(x)+f\left(v_{0}(x)\right) & >0 \\
v_{0}^{\prime \prime}(x)+g\left(u_{0}(x)\right) & >0 \\
u_{0}^{\prime}(x) & >0 \\
v_{0}^{\prime}(x) & >0
\end{aligned}
$$

The solutions of the System (2) are said to quench simultaneously in a finite time if there exists $T_{q}<\infty$ such that

$$
\max _{0 \leq x \leq 1}\{u(x, t)\} \rightarrow c^{-} \text {and } \max _{0 \leq x \leq 1}\{v(x, t)\} \rightarrow c^{-} \text {as } t \rightarrow T_{q}^{-} .
$$

However, if either $\max _{0 \leq x \leq 1}\{u(x, t)\} \rightarrow c^{-}$or $\max _{0 \leq x \leq 1}\{v(x, t)\} \rightarrow c^{-}$as $t \rightarrow T_{q}^{-}$, we say that the quenching is non-simultaneous. The time $T_{q}$ is called the quenching time of (2).

This article is organized as follows. In Section 2, we prove the comparison principles for heat inequlity and system of coupled heat inequalities involving the Neumann boundary conditions. In Section 3, we prove the existence of solutions to our problem. In Section 4, we determine conditions under which we are guaranteed the queching in a finite time. We also prove that the time derivatives become unbounded when quenching occurs. The quenching set is also provided. In Section 5, we characterize when simultaneous or non-simultaneous quenching are possible. We also give the quenching rates when non-simultaneous quenching occurs. Section 6 closes with disscussion and conclusion.

\section{Comparison Principles}

The aim of this section is to establish two comparison principles. We modify the idea of Reference [5] to obtain the proof of Theorem 1. We note that the boundary conditions of the problem in Theorem 1 are different from those of Reference [5]. 
Theorem 1. Let $\Theta(x, t) \in C([0,1] \times[0, T]) \cap C^{2,1}((0,1) \times(0, T))$ be a function satisfying

$$
\left.\begin{array}{ll}
\Theta_{t}(x, t)-\Theta_{x x}(x, t) \geq 0, & (x, t) \in(0,1) \times(0, T), \\
\Theta_{x}(0, t) \leq 0 \text { and } \Theta_{x}(1, t) \geq 0, & t \in[0, T], \\
\Theta(x, 0) \geq 0, & x \in[0,1] .
\end{array}\right\}
$$

Then, $\Theta(x, t) \geq 0$ in $[0,1] \times[0, T]$.

Proof. Let $\lambda>0$ and $n \in \mathbb{N}$. Define $\Psi(x, t)=e^{-\lambda t} \Theta(x, t)$ and $\Psi_{n}(x, t)=\Psi(x, t)+\frac{1}{n}$ in $[0,1] \times[0, T]$. For $(x, t) \in(0,1) \times(0, T)$, we have

$$
\begin{aligned}
\frac{\partial}{\partial t} \Psi_{n}(x, t) & =e^{-\lambda t} \Theta_{t}(x, t)-\lambda e^{-\lambda t} \Theta(x, t) \\
& \geq e^{-\lambda t} \Theta_{x x}(x, t)-\lambda \Psi(x, t) \\
& =\frac{\partial^{2}}{\partial x^{2}} \Psi_{n}(x, t)-\lambda \Psi_{n}(x, t)+\frac{\lambda}{n} \\
& >\frac{\partial^{2}}{\partial x^{2}} \Psi_{n}(x, t)-\lambda \Psi_{n}(x, t),
\end{aligned}
$$

and

$$
\frac{\partial}{\partial x} \Psi_{n}(x, t)=\frac{\partial}{\partial x} \Psi(x, t)=e^{-\lambda t} \frac{\partial}{\partial x} \Theta(x, t) .
$$

By the boundary and initial conditions in Equation (7), we have $\frac{\partial}{\partial x} \Psi_{n}(0, t) \leq 0, \frac{\partial}{\partial x} \Psi_{n}(1, t) \geq 0$, $t \in[0, T]$ and $\Psi_{n}(x, 0) \geq 0, x \in[0,1]$.

For each $n \in \mathbb{N}$, suppose that $\Psi_{n}(x, t)$ has a negative minimum $m$ at some point $\left(x_{k}, t_{k}\right) \in$ $[0,1] \times[0, T]$.

If $\left(x_{k}, t_{k}\right) \in[0,1] \times\{0\}$, then, by the initial condition in $(7), \Psi_{n}(x, 0) \geq 0$ for $x \in[0,1]$. This gives a contradiction.

$$
\begin{aligned}
& \text { If }\left(x_{k}, t_{k}\right) \in(0,1) \times(0, T) \text {, then } \frac{\partial}{\partial t} \Psi_{n}\left(x_{k}, t_{k}\right)=0 \text { and } \frac{\partial^{2}}{\partial x^{2}} \Psi_{n}\left(x_{k}, t_{k}\right) \geq 0 \text {. This gives } \\
& \lambda \Psi_{n}\left(x_{k}, t_{k}\right) \geq \lambda \Psi_{n}\left(x_{k}, t_{k}\right)-\frac{\partial^{2}}{\partial x^{2}} \Psi_{n}\left(x_{k}, t_{k}\right)=\frac{\partial}{\partial t} \Psi_{n}\left(x_{k}, t_{k}\right)-\frac{\partial^{2}}{\partial x^{2}} \Psi_{n}\left(x_{k}, t_{k}\right)+\lambda \Psi_{n}\left(x_{k}, t_{k}\right)>0,
\end{aligned}
$$

which implies that $\Psi_{n}\left(x_{k}, t_{k}\right)>0$. We have a contradiction.

If $\left(x_{k}, t_{k}\right) \in\{0\} \times(0, T]$, then there exists a neighborhood $H$ of $\{0\} \times(0, T]$ such that $\Psi_{n}(x, t)>m$ for all $(x, t) \in H$. Then, by Theorem 14 [6] (p. 47), $\frac{\partial}{\partial x} \Psi_{n}(0, t)>0$ for all $t \in(0, T]$. We have a contradiction.

If $\left(x_{k}, t_{k}\right) \in\{1\} \times(0, T]$, then there exists a neighborhood $H$ of $\{1\} \times(0, T]$ such that $\Psi_{n}(x, t)>m$ for all $(x, t) \in H$. Then, by Theorem 14 [6] (p. 47), $\frac{\partial}{\partial x} \Psi_{n}(1, t)<0$ for all $t \in(0, T]$, where we have, again, a contradiction.

Hence, $\Psi_{n}(x, t) \geq 0$ in $[0,1] \times[0, T]$. By letting $n \rightarrow \infty$, we can conclude that $\Psi(x, t) \geq 0$ in $[0,1] \times[0, T]$. Therefore, $\Theta(x, t) \geq 0$ in $[0,1] \times[0, T]$. 
Theorem 2. Let $F, G$ be non-negative functions and $\Theta(x, t), \Psi(x, t) \in C([0,1] \times[0, T]) \cap C^{2,1}((0,1) \times(0, T))$ be functions satisfying

$$
\left.\begin{array}{ll}
\Theta_{t}(x, t)-\Theta_{x x}(x, t)+F(x, t) \Psi(x, t) \geq 0, & (x, t) \in(0,1) \times(0, T), \\
\Psi_{t}(x, t)-\Psi_{x x}(x, t)+G(x, t) \Theta(x, t) \geq 0, & (x, t) \in(0,1) \times(0, T), \\
\Theta_{x}(0, t) \leq 0 \text { and } \Theta_{x}(1, t) \geq 0, & t \in[0, T], \\
\Psi_{x}(0, t) \leq 0 \text { and } \Psi_{x}(1, t) \geq 0, & t \in[0, T], \\
\Theta(x, 0) \geq 0 \text { and } \Psi(x, 0) \geq 0, & x \in[0,1] .
\end{array}\right\}
$$

Then, $\Theta(x, t) \geq 0$ and $\Psi(x, t) \geq 0$ in $[0,1] \times[0, T]$.

Proof. Define $\Phi(x, t)=e^{-2 t-x^{2}} \Theta(x, t)$ and $\Xi(x, t)=e^{-2 t-x^{2}} \Psi(x, t)$ in $[0,1] \times[0, T]$. Suppose that $\Phi(x, t)$ has a negative minimum $m$ at some point $\left(x_{k}, t_{k}\right) \in[0,1] \times[0, T]$. Without loss of generality, let $m=\Phi\left(x_{k}, t_{k}\right) \leq \min _{(x, t) \in[0,1] \times[0, T]} \Xi(x, t)$.

If $\left(x_{k}, t_{k}\right) \in[0,1] \times\{0\}$, then, by the initial condition in Equation (8), we must have $\Phi(x, 0) \geq 0$ for all $x \in[0,1]$. This directs to a contradiction.

If $\left(x_{k}, t_{k}\right) \in(0,1) \times(0, T)$, then for all $(x, t) \in(0,1) \times(0, T)$, we have

$$
\Phi_{t}(x, t)=e^{-2 t-x^{2}} \Theta_{t}(x, t)-2 e^{-2 t-x^{2}} \Theta(x, t)
$$

and

$$
\Phi_{x x}(x, t)=e^{-2 t-x^{2}} \Theta_{x x}(x, t)-4 x e^{-2 t-x^{2}} \Theta_{x}(x, t)+4 x^{2} e^{-2 t-x^{2}} \Theta(x, t)-2 e^{-2 t-x^{2}} \Theta(x, t) .
$$

Thus, for all $(x, t) \in(0,1) \times(0, T)$,

$$
\Phi_{x x}(x, t)+4 x \Phi_{x}(x, t)-\Phi_{t}(x, t)+4 x^{2} \Phi(x, t)+F(x, t) \Xi(x, t) \geq 0
$$

and

$$
\Xi_{x x}(x, t)+4 x \Xi_{x}(x, t)-\Xi_{t}(x, t)+4 x^{2} \Xi(x, t)+G(x, t) \Phi(x, t) \geq 0 .
$$

Since $\Phi\left(x_{k}, t_{k}\right)=m, \Phi(x, t)-m>0$ and $\Xi(x, t)-m>0$ on the boundaries $(x, t) \in\{0,1\} \times(0, T]$. By the initial conditions in Equation (8), we have $\Phi(x, 0)-m>0$ and $\Xi(x, 0)-m>0$, for all $x \in[0,1]$. Then, by Theorem 15 [7], (p. 191) $\Phi(x, t)>m$ and $\Xi(x, t)>m$ for all $(x, t) \in(0,1) \times(0, T)$. This gives a contradiction.

If $\left(x_{k}, t_{k}\right) \in\{0\} \times(0, T]$, then there exists a neighborhood $H$ of $\{0\} \times(0, T]$ such that $\Phi(x, t)>m$ for all $(x, t) \in H$. Thus, by Theorem 15 [7] (p. 191), $\frac{\partial}{\partial x} \Phi(0, t)>0$ for all $t \in(0, T]$. However, it can be directly calculated that $\Phi_{x}(0, t)=e^{-2 t} \Theta_{x}(0, t) \leq 0$ for all $t \in(0, T]$. We have a contradiction.

If $\left(x_{k}, t_{k}\right) \in\{1\} \times(0, T]$, then there exists a neighborhood $H$ of $\{1\} \times(0, T]$ such that $\Phi(x, t)>m$ for all $(x, t) \in H$. Thus, by Theorem 15 [7] (p. 191), $\frac{\partial}{\partial x} \Phi(1, t)<0$ for all $t \in(0, T]$. However, it can be directly calculated that $\Phi_{x}(1, t)=e^{-2 t-1}\left(\Theta_{x}(1, t)-2 \Theta(1, t)\right)>0$ for all $t \in(0, T]$. We have, again, a contradiction.

Therefore, $\Phi(x, t) \geq 0$ and $\Xi(x, t) \geq 0$ in $[0,1] \times[0, T]$, which implies $\Theta(x, t) \geq 0$ and $\Psi(x, t) \geq 0$ in $[0,1] \times[0, T]$.

\section{Existence of Solutions}

In this section, we use the technique of upper and lower solutions to investigate the existence result of our problem. 
Definition 1. A pair of functions $(\widetilde{u}, \widetilde{v})$ is called an upper solution of Equation (2) for $(x, t) \in[0,1] \times[0, T]$ if $\widetilde{u}, \widetilde{v} \in C([0,1] \times[0, T]) \cap C^{2,1}((0,1) \times(0, T))$ and $(\widetilde{u}, \widetilde{v})$ satisfies

$$
\begin{array}{lrl}
\widetilde{u}_{t}(x, t) \geq \widetilde{u}_{x x}(x, t)+f(\widetilde{v}(x, t)), & (x, t) \in(0,1) \times(0, T), \\
\widetilde{v}_{t}(x, t) \geq \widetilde{v}_{x x}(x, t)+g(\widetilde{u}(x, t)), & (x, t) \in(0,1) \times(0, T), \\
\widetilde{u}_{x}(0, t)=\widetilde{u}_{x}(1, t)=0 \text { and } \widetilde{v}_{x}(0, t)=\widetilde{v}_{x}(1, t)=0, & t \in[0, T], \\
\widetilde{u}(x, 0) \geq u_{0}(x) \text { and } \widetilde{v}(x, 0) \geq v_{0}(x), & x \in[0,1] .
\end{array}
$$

Similarly, a lower solution $(\widehat{u}, \widehat{v})$ of $(2)$ is defined by reversing all inequalities in Definition 1.

We modify the proof of Lemma 2.1 in Reference [8] to obtain Lemma 1. We note here that the forcing terms appeared in Lemma 1 are more general than those in Reference [8].

Lemma 1. Let $(\widetilde{u}, \widetilde{v})$ and $(\widehat{u}, \widehat{v})$ be a positive upper solution and a non-negative lower solution of the System (2) for $(x, t) \in[0,1] \times[0, T]$, respectively. Then, $\widetilde{u} \geq \widehat{u}$ and $\widetilde{v} \geq \widehat{v}$ in $[0,1] \times[0, T]$. In particular, if $\left(u^{*}, v^{*}\right)$ is a solution of (2), then $\widetilde{u} \geq u^{*} \geq \widehat{u}$ and $\widetilde{v} \geq v^{*} \geq \widehat{v}$ in $[0,1] \times[0, T]$.

Proof. Let $W(x, t)=\widetilde{u}(x, t)-\widehat{u}(x, t)$ and $Z(x, t)=\widetilde{v}(x, t)-\widehat{v}(x, t)$ in $[0,1] \times[0, T]$. Then,

$$
W_{t}(x, t)=\widetilde{u}_{t}(x, t)-\widehat{u}_{t}(x, t) \geq \widetilde{u}_{x x}(x, t)-\widehat{u}_{x x}(x, t)+f(\widetilde{v}(x, t))-f(\widehat{v}(x, t))
$$

for all $(x, t) \in(0,1) \times(0, T)$. This gives

$$
W_{t}(x, t) \geq W_{x x}(x, t)+f(\widetilde{v}(x, t))-f(\widehat{v}(x, t))=W_{x x}(x, t)+a(x, t) Z(x, t)
$$

for all $(x, t) \in(0,1) \times(0, T)$, where $a(x, t)=\frac{f(\widetilde{v}(x, t))-f(\widehat{v}(x, t))}{\widetilde{v}(x, t)-\widehat{v}(x, t)}$ if $\widetilde{v}(x, t) \neq \widehat{v}(x, t)$; otherwise, $a(x, t)=0$. Similarly, for $(x, t) \in(0,1) \times(0, T)$, we have

$$
Z_{t}(x, t)=\widetilde{v}_{t}(x, t)-\widehat{v}_{t}(x, t) \geq \widetilde{v}_{x x}(x, t)-\widehat{v}_{x x}(x, t)+g(\widetilde{u}(x, t))-g(\widehat{u}(x, t)) .
$$

This gives

$$
Z_{t}(x, t) \geq Z_{x x}(x, t)+g(\widetilde{u}(x, t))-g(\widehat{u}(x, t))=Z_{x x}(x, t)+b(x, t) W(z, t)
$$

for all $(x, t) \in(0,1) \times(0, T)$, where $b(x, t)=\frac{g(\widetilde{u}(x, t))-g(\widehat{u}(x, t))}{\widetilde{u}(x, t)-\widehat{u}(x, t)}$ if $\widetilde{u}(x, t) \neq \widehat{u}(x, t)$; otherwise, $b(x, t)=0$. At the boundaries $x=0$ and $x=1$, we have

$$
\begin{array}{r}
W_{x}(0, t)=\widetilde{u}_{x}(0, t)-\widehat{u}_{x}(0, t)=0 \text { and } W_{x}(1, t)=\widetilde{u}_{x}(1, t)-\widehat{u}_{x}(1, t)=0, \\
Z_{x}(0, t)=\widetilde{v}_{x}(0, t)-\widehat{v}_{x}(0, t)=0 \text { and } Z_{x}(1, t)=\widetilde{v}_{x}(1, t)-\widehat{v}_{x}(1, t)=0 .
\end{array}
$$

By the initial conditions of the upper and lower solutions, we obtain

$$
\begin{array}{r}
W(x, 0)=\widetilde{u}(x, 0)-\widehat{u}(x, 0) \geq u_{0}(x)-u_{0}(x)=0, \\
Z(x, 0)=\widetilde{v}(x, 0)-\widehat{v}(x, 0) \geq v_{0}(x)-v_{0}(x)=0
\end{array}
$$

for all $x \in[0,1]$. By Theorem 2, we have $W(x, t) \geq 0$ and $Z(x, t) \geq 0$ for $(x, t) \in[0,1] \times[0, T]$, which implies $\widetilde{u}(x, t) \geq \widehat{u}(x, t)$ and $\widetilde{v}(x, t) \geq \widehat{v}(x, t)$ for all $(x, t) \in[0,1] \times[0, T]$.

Next, let us define two monotone sequences of functions $\left\{\bar{u}^{(k)}, \bar{v}^{(k)}\right\}$ and $\left\{\underline{u}^{(k)}, \underline{v}^{(k)}\right\}$ for $k \in \mathbb{N}$ which we refer them as the maximal and the minimal sequences, respectively, where the initial guesses 
are $\left(\bar{u}^{(0)}, \bar{v}^{(0)}\right)=(\widetilde{u}, \widetilde{v})$ and $\left(\underline{u}^{(0)}, \underline{v}^{(0)}\right)=(\widehat{u}, \widehat{v})$ and those maximal and minimal sequences satisfy the linear problem (9) and the boundary and initial conditions thereafter.

$$
\left.\begin{array}{l}
u_{t}^{(k)}(x, t)=u_{x x}^{(k)}(x, t)+f\left(v^{(k-1)}(x, t)\right),(x, t) \in(0,1) \times(0, T), \\
v_{t}^{(k)}(x, t)=v_{x x}^{(k)}(x, t)+g\left(u^{(k-1)}(x, t)\right),(x, t) \in(0,1) \times(0, T),
\end{array}\right\},
$$

subject to the boundary conditions

$$
u_{x}^{(k)}(0, t)=u_{x}^{(k)}(1, t)=0 \text { and } v_{x}^{(k)}(0, t)=v_{x}^{(k)}(1, t)=0, t \in(0, T]
$$

and the initial conditions

$$
u^{(k)}(x, 0)=u_{0}(x) \text { and } v^{(k)}(x, 0)=v_{0}(x), x \in[0,1],
$$

where $k \in \mathbb{N}$.

Lemma 2. The two sequences $\left\{\bar{u}^{(k)}, \bar{v}^{(k)}\right\}$ and $\left\{\underline{u}^{(k)}, \underline{v}^{(k)}\right\}$ possess the monotone property

$$
(\widehat{u}, \widehat{v}) \leq\left(\underline{u}^{(k)}, \underline{v}^{(k)}\right) \leq\left(\underline{u}^{(k+1)}, \underline{v}^{(k+1)}\right) \leq\left(\bar{u}^{(k+1)}, \bar{v}^{(k+1)}\right) \leq\left(\bar{u}^{(k)}, \bar{v}^{(k)}\right) \leq(\widetilde{u}, \widetilde{v})
$$

for all $(x, t) \in[0,1] \times[0, T]$ and $k \in \mathbb{N}$. Here, $(a, b) \leq(c, d)$ implies $a \leq c$ and $b \leq d$.

Proof. Let $W(x, t)=\widetilde{u}(x, t)-\bar{u}^{(1)}(x, t)$ and $Z(x, t)=\widetilde{v}(x, t)-\bar{v}^{(1)}(x, t)$ in $[0,1] \times[0, T]$. We have by Equation (9) and $\left(\bar{u}^{(0)}, \bar{v}^{(0)}\right)=(\widetilde{u}, \widetilde{v})$ that, for $(x, t) \in(0,1) \times(0, T)$,

$$
\begin{aligned}
W_{t}(x, t)-W_{x x}(x, t) & =\widetilde{u}_{t}(x, t)-\widetilde{u}_{x x}(x, t)-f(\widetilde{v}(x, t)) \geq 0, \\
Z_{t}(x, t)-Z_{x x}(x, t) & =\widetilde{v}_{t}(x, t)-\widetilde{v}_{x x}(x, t)-g(\widetilde{u}(x, t)) \geq 0 .
\end{aligned}
$$

Since $\widetilde{u}_{x}(0, t)=\widetilde{u}_{x}(1, t)=\bar{u}_{x}^{(1)}(0, t)=\bar{u}_{x}^{(1)}(1, t)=0$ and $\widetilde{v}_{x}(0, t)=\widetilde{v}_{x}(1, t)=\bar{v}_{x}^{(1)}(0, t)=$ $\bar{v}_{x}^{(1)}(1, t)=0$ for $t \in(0, T]$, we have

$$
W_{x}(0, t)=W_{x}(1, t)=0 \text { and } Z_{x}(0, t)=Z_{x}(1, t)=0
$$

for $t \in(0, T]$. Since $\widetilde{u}(x, 0) \geq u_{0}(x)=\bar{u}^{(1)}(x, 0)$ and $\widetilde{v}(x, 0) \geq v_{0}(x)=\bar{v}^{(1)}(x, 0)$ for $x \in[0,1]$, we have

$$
W(x, 0)=\widetilde{u}(x, 0)-u_{0}(x) \geq 0 \text { and } Z(x, 0)=\widetilde{v}(x, 0)-v_{0}(x) \geq 0
$$

for $x \in[0,1]$. By Theorem 1 , we have $W(x, t) \geq 0$ and $Z(x, t) \geq 0$ for $(x, t) \in[0,1] \times[0, T]$. This gives

$$
\bar{u}^{(1)}(x, t) \leq \widetilde{u}(x, t) \text { and } \bar{v}^{(1)}(x, t) \leq \widetilde{v}(x, t) \text { for }(x, t) \in[0,1] \times[0, T] .
$$

Similarly, using the property of a lower solution and Theorem 1 we obtain

$$
\underline{u}^{(1)}(x, t) \geq \widehat{u}(x, t) \text { and } \underline{v}^{(1)}(x, t) \geq \widehat{v}(x, t) \text { for }(x, t) \in[0,1] \times[0, T] .
$$

The next step is obtained by the mathematical induction. Let

$$
W^{(1)}(x, t)=\bar{u}^{(1)}(x, t)-\underline{u}^{(1)}(x, t) \text { and } Z^{(1)}(x, t)=\bar{v}^{(1)}(x, t)-\underline{v}^{(1)}(x, t)
$$


for all $(x, t) \in[0,1] \times[0, T]$. Then, for all $(x, t) \in(0,1) \times(0, T)$, we have

$$
\begin{aligned}
W_{t}^{(1)}(x, t) & =\bar{u}_{t}^{(1)}(x, t)-\underline{u}_{t}^{(1)}(x, t)=\bar{u}_{x x}^{(1)}(x, t)+f(\widetilde{v}(x, t))-\underline{u}_{x x}^{(1)}(x, t)-f(\widehat{v}(x, t)), \\
Z_{t}^{(1)}(x, t) & =\bar{v}_{t}^{(1)}(x, t)-\underline{v}_{t}^{(1)}(x, t)=\bar{v}_{x x}^{(1)}(x, t)+g(\widetilde{u}(x, t))-\underline{v}_{x x}^{(1)}(x, t)-g(\widehat{u}(x, t))
\end{aligned}
$$

Therefore, for $(x, t) \in(0,1) \times(0, T)$, we have

$$
W_{t}^{(1)}(x, t)-W_{x x}^{(1)}(x, t)=f(\widetilde{v}(x, t))-f(\widehat{v}(x, t)) \geq 0
$$

and

$$
Z_{t}^{(1)}(x, t)-Z_{x x}^{(1)}(x, t)=g(\widetilde{u}(x, t))-g(\widehat{u}(x, t)) \geq 0 .
$$

Since $\bar{u}_{x}^{(1)}(0, t)=\underline{u}_{x}^{(1)}(0, t)=\bar{u}_{x}^{(1)}(1, t)=\underline{u}_{x}^{(1)}(1, t)=0$ and $\bar{v}_{x}^{(1)}(0, t)=\underline{v}_{x}^{(1)}(0, t)=\bar{v}_{x}^{(1)}(1, t)=\underline{v}_{x}^{(1)}(1, t)=0$ for $t \in(0, T]$, we have

$$
W_{x}^{(1)}(0, t)=W_{x}^{(1)}(1, t)=0 \text { and } Z_{x}^{(1)}(0, t)=Z_{x}^{(1)}(1, t)=0
$$

for $t \in(0, T]$. Since $\bar{u}^{(1)}(x, 0)=u_{0}(x)=\underline{u}^{(1)}(x, 0)$ and $\bar{v}^{(1)}(x, 0)=v_{0}(x)=\underline{v}^{(1)}(x, 0)$ for $x \in[0,1]$, we have

$$
W^{(1)}(x, 0)=0 \text { and } Z^{(1)}(x, 0)=0
$$

for all $x \in[0,1]$. By Theorem $1, W^{(1)}(x, t) \geq 0$ and $Z^{(1)}(x, t) \geq 0$ for $(x, t) \in[0,1] \times[0, T]$. Thus, $\underline{u}^{(1)}(x, t) \leq \bar{u}^{(1)}(x, t)$ and $\underline{v}^{(1)}(x, t) \leq \bar{v}^{(1)}(x, t)$ for all $(x, t) \in[0,1] \times[0, T]$. Therefore, for all $(x, t) \in[0,1] \times[0, T]$, we obtain

$$
(\widehat{u}, \widehat{v}) \leq\left(\underline{u}^{(1)}, \underline{v}^{(1)}\right) \leq\left(\bar{u}^{(1)}, \bar{v}^{(1)}\right) \leq(\widetilde{u}, \widetilde{v} .)
$$

Next, let $k \geq 2$ be an integer and assume

$$
\left(\underline{u}^{(k-1)}, \underline{v}^{(k-1)}\right) \leq\left(\underline{u}^{(k)}, \underline{v}^{(k)}\right) \leq\left(\bar{u}^{(k)}, \bar{v}^{(k)}\right) \leq\left(\bar{u}^{(k-1)}, \bar{v}^{(k-1)}\right)
$$

for all $(x, t) \in[0,1] \times[0, T]$.

Let $W^{(k)}(x, t)=\bar{u}^{(k)}(x, t)-\bar{u}^{(k+1)}(x, t)$ and $Z^{(k)}(x, t)=\bar{v}^{(k)}(x, t)-\bar{v}^{(k+1)}(x, t)$ in $[0,1] \times[0, T]$.

Then,

$$
\begin{aligned}
W_{t}^{(k)}(x, t) & =\bar{u}_{t}^{(k)}(x, t)-\bar{u}_{t}^{(k+1)}(x, t)=\bar{u}_{x x}^{(k)}(x, t)+f\left(\bar{v}^{(k-1)}(x, t)\right)-\bar{u}_{x x}^{(k+1)}(x, t)-f\left(\bar{v}^{(k)}(x, t)\right), \\
Z_{t}^{(k)}(x, t) & =\bar{v}_{t}^{(k)}(x, t)-\bar{v}_{t}^{(k+1)}(x, t)=\bar{v}_{x x}^{(k)}(x, t)+g\left(\bar{u}^{(k-1)}(x, t)\right)-\bar{v}_{x x}^{(k+1)}(x, t)-g\left(\bar{u}^{(k)}(x, t)\right),
\end{aligned}
$$

and

$$
W_{x x}^{(k)}(x, t)=\bar{u}_{x x}^{(k)}(x, t)-\bar{u}_{x x}^{(k+1)}(x, t) \text { and } Z_{x x}^{(k)}(x, t)=\bar{v}_{x x}^{(k)}(x, t)-\bar{v}_{x x}^{(k+1)}(x, t) .
$$

By the induction hypothesis, $f$ and $g$ being increasing functions, we can conclude that, for $(x, t) \in(0,1) \times(0, T)$,

$$
\begin{aligned}
W_{t}^{(k)}(x, t)-W_{x x}^{(k)}(x, t) & =f\left(\bar{v}^{(k-1)}(x, t)\right)-f\left(\bar{v}^{(k)}(x, t)\right) \geq 0, \\
Z_{t}^{(k)}(x, t)-Z_{x x}^{(k)}(x, t) & =g\left(\bar{u}^{(k-1)}(x, t)\right)-g\left(\bar{u}^{(k))}(x, t)\right) \geq 0 .
\end{aligned}
$$


Since $\bar{u}_{x}^{(k)}(0, t)=\bar{u}_{x}^{(k)}(1, t)=\bar{u}_{x}^{(k+1)}(0, t)=\bar{u}_{x}^{(k+1)}(1, t)=0$ and $\bar{v}_{x}^{(k)}(0, t)=\bar{v}_{x}^{(k)}(1, t)=\bar{v}_{x}^{(k+1)}(0, t)=\bar{v}_{x}^{(k+1)}(1, t)=0$ for $t \in(0, T]$, we have

$$
W_{x}^{(k)}(0, t)=W_{x}^{(k)}(1, t)=0 \text { and } Z_{x}^{(k)}(0, t)=Z_{x}^{(k)}(1, t)=0
$$

for $t \in(0, T]$. Since $\bar{u}^{(k)}(x, 0)=u_{0}(x)=\bar{u}^{(k+1)}(x, 0)$ and $\bar{v}^{(k)}(x, 0)=v_{0}(x)=\bar{v}^{(k+1)}(x, 0)$ for $x \in[0,1]$, we have for $x \in[0,1]$ that

$$
W^{(k)}(x, t)=0 \text { and } Z^{(k)}(x, 0)=0
$$

By Theorem $1, W^{(k)}(x, t) \geq 0$ and $Z^{(k)}(x, t) \geq 0$ for all $(x, t) \in[0,1] \times[0, T]$. This gives $\bar{u}^{(k+1)}(x, t) \leq \bar{u}^{(k)}(x, t)$ and $\bar{v}^{(k+1)}(x, t) \leq \bar{v}^{(k)}(x, t)$ for all $(x, t) \in[0,1] \times[0, T]$. By using a similar argument, we obtain $\underline{u}^{(k+1)}(x, t) \geq \underline{u}^{(k)}(x, t)$ and $\underline{v}^{(k+1)}(x, t) \geq \underline{v}^{(k)}(x, t)$ for all $(x, t) \in[0,1] \times[0, T]$, also $\bar{u}^{(k+1)}(x, t) \geq \underline{u}^{(k+1)}(x, t)$ and $\bar{v}^{(k+1)}(x, t) \geq \underline{v}^{(k+1)}(x, t)$ for all $(x, t) \in[0,1] \times[0, T]$. The result follows from the mathematical induction.

We have from Lemma 2 that the sequences $\left\{\bar{u}^{(k)}\right\}$ and $\left\{\bar{v}^{(k)}\right\}$ are monotone decreasing and are bounded from below, while the sequence $\left\{\underline{u}^{(k)}\right\}$ and $\left\{\underline{v}^{(k)}\right\}$ are monotone increasing and are bounded from above. Therefore, the pointwise limits of sequences exist and we arrive at the conclusion that the solutions $u$ and $v$ to the System (2) exist.

\section{Finite-Time Quenching of $(u, v)$ and Blow-Up of $\left(u_{t}, v_{t}\right)$}

In this section, we provide the sufficient conditions to guarantee quenching in a finite time of the System (2). First, we prove that the solutions $u$ and $v$ are increasing in space and increasing in time.

\section{Lemma 3.}

(i) If the initial conditions satisfy (5) and (6), then $u_{x}(x, t) \geq 0$ and $v_{x}(x, t) \geq 0$ for $(x, t) \in[0,1] \times[0, T)$.

(ii) If the initial conditions satisfy (3) and (4), then $u_{t}(x, t) \geq 0$ and $v_{t}(x, t) \geq 0$ for $(x, t) \in[0,1] \times[0, T)$.

\section{Proof.}

(i) Assume that $u_{0}(x)$ and $v_{0}(x)$ satisfy (5) and (6), respectively. For any fixed $\tau \in(0, T)$, let us define $W(x, t)=u_{x}(x, t)$ and $Z(x, t)=v_{x}(x, t)$ in $[0,1] \times[0, \tau]$. We have by (2) that

$$
\begin{aligned}
W_{t}(x, t) & =\left(u_{t}\right)_{x}(x, t)=u_{x x x}(x, t)+f^{\prime}(v(x, t)) v_{x}(x, t)=W_{x x}(x, t)+f^{\prime}(v(x, t)) Z(x, t), \\
Z_{t}(x, t) & =\left(v_{t}\right)_{x}(x, t)=v_{x x x}(x, t)+g^{\prime}(u(x, t)) u_{x}(x, t)=Z_{x x}(x, t)+g^{\prime}(u(x, t)) W(x, t),
\end{aligned}
$$

for all $(x, t) \in(0,1) \times(0, T)$. Since $u_{x}(0, t)=u_{x}(1, t)=0$ and $v_{x}(0, t)=v_{x}(1, t)=0$, we have

$$
W(0, t)=W(1, t)=0 \text { and } Z(0, t)=Z(1, t)=0
$$

for $t \in(0, T]$. By Equations (5) and (6), $W(x, 0)=u_{0}^{\prime}(x)>0$ and $Z(x, 0)=v_{0}^{\prime}(x)>0, x \in[0,1]$. Then, by Theorem 15 [7] (p. 191), $u_{x}(x, t)=W(x, t) \geq 0$ and $v_{x}(x, t)=Z(x, t) \geq 0$ for $(x, t) \in$ $[0,1] \times[0, T)$.

(ii) Assume (3) and (4) hold. For any fixed $\tau \in(0, T)$, define $W(x, t)=u_{t}(x, t)$ and $Z(x, t)=v_{t}(x, t)$ in $[0,1] \times[0, \tau]$. We have by $(2)$ that

$$
\begin{aligned}
& W_{t}(x, t)=\left(u_{t}\right)_{t}(x, t)=u_{x x t}(x, t)+f^{\prime}(v(x, t)) v_{t}(x, t)=W_{x x}(x, t)+f^{\prime}(v(x, t)) Z(x, t), \\
& Z_{t}(x, t)=\left(v_{t}\right)_{t}(x, t)=v_{x x t}(x, t)+g^{\prime}(u(x, t)) u_{t}(x, t)=Z_{x x}(x, t)+g^{\prime}(u(x, t)) W(x, t),
\end{aligned}
$$


for all $(x, t) \in(0,1) \times(0, T)$. Differentiating $W(x, t)$ and $Z(x, t)$ with respect to $x$, we have by Equation (2) that

$$
\begin{aligned}
W_{x}(x, t) & =\left(u_{t}\right)_{x}(x, t)=u_{x x x}(x, t)+f^{\prime}(v(x, t)) v_{x}(x, t), \\
Z_{x}(x, t) & =\left(v_{t}\right)_{x}(x, t)=v_{x x x}(x, t)+g^{\prime}(u(x, t)) u_{x}(x, t) .
\end{aligned}
$$

Since $u_{x}(0, t)=u_{x}(1, t)=0$ and $v_{x}(0, t)=v_{x}(1, t)=0$, we have

$$
W_{x}(0, t)=W_{x}(1, t)=0 \text { and } Z_{x}(0, t)=Z_{x}(1, t)=0,
$$

for $t \in(0, \tau]$. By Equations (3) and (4), $W(x, 0)=u_{0}^{\prime \prime}(x)+f\left(v_{0}(x)\right)>0$ and $Z(x, 0)=v_{0}^{\prime \prime}(x)+g\left(u_{0}(x)\right)>0$. By Theorem $2, u_{t}(x, t)=W(x, t) \geq 0$ and $v_{t}(x, t)=Z(x, t) \geq 0$ for all $(x, t) \in[0,1] \times[0, T)$.

By modifying the proof of Theorem 2 of Reference [9] and extending the forcing terms in their proof to more general forcing functions, the result of quenching in a finite time can be established.

\section{Theorem 3.}

(i) If the initial conditions satisfy Equation (3), then u quenches in a finite time.

(ii) If the initial conditions satisfy Equation (4), then $v$ quenches in a finite time.

Proof. We will give the proof of (i). The proof of (ii) can be done in a similar manner. Assume that $u_{0}(x)$ and $v_{0}(x)$ satisfy (3). Then, $\int_{0}^{1} f\left(v_{0}(x)\right)>0$. Define $m(t)=\int_{0}^{1}(c-u(x, t)) d x, t \in[0, T]$. We have by Leibniz's rule, (2), the boundary conditions, and $f$ being an increasing function that

$$
m^{\prime}(t)=-\int_{0}^{1} u_{t}(x, t) d x=-\int_{0}^{1}\left(u_{x x}(x, t)+f(v(x, t))\right) d x=-\int_{0}^{1} f(v(x, t)) d x \leq-\int_{0}^{1} f\left(v_{0}(x)\right) d x .
$$

Integrating Equation (10) with respect to $t$ from 0 to $t$, we obtain

$$
m(t) \leq m(0)-t \int_{0}^{1} f\left(v_{0}(x)\right) d x, t \in[0, T] .
$$

From (11), there exists a finite time $t=T_{q}$ such that $m\left(T_{q}\right)=0$. Therefore, $u(x, t)$ quenches in a finite time.

\section{Theorem 4.}

(i) If the initial conditions satisfy Equations (3) and (5), then $x=1$ is the only quenching point of $u(x, t)$.

(ii) If the initial conditions satisfy Equations (4) and (6), then $x=1$ is the only quenching point of $v(x, t)$.

Proof. We will give the proof of (i). The proof of (ii) can be done by using a similar argument. For any fixed $b_{1} \in(0,1), b_{2} \in\left(b_{1}, 1\right)$ and $\tau \in(0, T)$. Let $\epsilon>0$ and define $G(x, t)=u_{x}(x, t)-\epsilon\left(x-b_{1}\right)$ in $\left[b_{1}, b_{2}\right] \times[0, \tau]$. Then,

$$
G_{t}(x, t)-G_{x x}(x, t)=f^{\prime}(v(x, t)) v_{x}(x, t) \geq 0 \text { for }(x, t) \in\left(b_{1}, b_{2}\right) \times(0, \tau) .
$$

Furthermore, if $\epsilon$ is small enough, then

$$
\begin{aligned}
& G\left(b_{1}, t\right)=u_{x}\left(b_{1}, t\right)>0 \text { and } G\left(b_{2}, t\right)=u_{x}\left(b_{2}, t\right)-\epsilon\left(b_{2}-b_{1}\right)>0 \text { for } t \in[0, \tau], \\
& G(x, 0)=u_{x}(x, 0)-\epsilon\left(x-b_{1}\right)>0 \text { for } x \in\left[b_{1}, b_{2}\right] .
\end{aligned}
$$


By Theorem 15 [7] (p. 191), we obtain

$$
u_{x}(x, t)>\epsilon\left(x-b_{1}\right) \text { for }(x, t) \in\left[b_{1}, b_{2}\right] \times[0, T) .
$$

Integrating (12) with respect to $x$ from $b_{1}$ to $b_{2}$, we have

$$
u\left(b_{1}, t\right)<u\left(b_{2}, t\right)-\frac{\epsilon\left(b_{2}-b_{1}\right)^{2}}{2} \text { for } t \in[0, T) .
$$

Therefore, $u(x, t)$ does not quench in $(0,1)$. Next, we have to show that $u(0, t)$ does not quench at $T_{q}$. Suppose $u(0, t) \rightarrow c$ as $t \rightarrow T_{q}$. Then, there exists $\delta>0$ such that $u_{x}\left(\eta, T_{q}\right)<0$ where $\eta \in(0, \delta)$. By Lemma 3 (ii), we have a contradiction. Therefore, $u(x, t)$ does not quench in $[0,1)$. The theorem is proved.

The next Lemma will be used to prove that the time-derivatives blow up at the quenching time.

Lemma 4. If the initial conditions satisfy Equations (3) and (4), then there exists $\delta>0$ such that

$$
u_{t}(x, t) \geq \delta f(v(x, t)), v_{t}(x, t) \geq \delta g(u(x, t)),(x, t) \in[0,1] \times(0, T) .
$$

Proof. For any fixed $\tau_{1} \in\left(0, \tau_{2}\right)$ and $\tau_{2} \in(0, T)$ define $W(x, t)=u_{t}(x, t)-\delta f(v(x, t))$ and $Z(x, t)=v_{t}(x, t)-\delta g(u(x, t))$ for $(x, t) \in[0,1] \times\left[\tau_{1}, \tau_{2}\right]$. Then,

$$
W_{t}(x, t)=u_{t t}(x, t)-\delta f^{\prime}(v(x, t)) v_{t}(x, t),
$$

and

$$
W_{x x}(x, t)=u_{t x x}(x, t)-\delta f^{\prime}(v(x, t)) v_{x x}(x, t)-\delta f^{\prime \prime}(v(x, t))\left(v_{x}(x, t)\right)^{2} .
$$

Therefore,

$$
\begin{aligned}
W_{t}(x, t)-W_{x x}(x, t) & =u_{t t}-\delta f^{\prime}(v) v_{x x}-\delta f^{\prime}(v) g(u)-u_{t x x}+\delta f^{\prime}(v) v_{x x}+\delta f^{\prime \prime}(v)\left(v_{x}\right)^{2} \\
& =u_{x x t}+f^{\prime}(v) v_{t}-\delta f^{\prime}(v) g(u)-u_{t x x}+\delta f^{\prime \prime}(v)\left(v_{x}\right)^{2} \\
& =f^{\prime}(v)\left(v_{t}-\delta g(u)\right)+\delta f^{\prime \prime}(v)\left(v_{x}\right)^{2} \\
& \geq f^{\prime}(v) Z(x, t)
\end{aligned}
$$

for $(x, t) \in(0,1) \times\left(\tau_{1}, \tau_{2}\right)$. Similarly, we have

$$
Z_{t}(x, t)=v_{t t}(x, t)-\delta g^{\prime}(u(x, t)) u_{t}(x, t)
$$

and

$$
Z_{x x}(x, t)=v_{t x x}(x, t)-\delta g^{\prime}(u(x, t)) u_{x x}(x, t)-\delta g^{\prime \prime}(u(x, t))\left(u_{x}(x, t)\right)^{2} .
$$

Therefore,

$$
\begin{aligned}
Z_{t}(x, t)-Z_{x x}(x, t) & =v_{t t}-\delta g^{\prime}(u) u_{x x}-\delta g^{\prime}(u) f(v)-v_{t x x}+\delta g^{\prime}(u) u_{x x}+\delta g^{\prime \prime}(u)\left(u_{x}\right)^{2} \\
& =v_{x x t}+g^{\prime}(u) u_{t}-\delta g^{\prime}(u) f(v)-v_{t x x}+\delta g^{\prime \prime}(u)\left(u_{x}\right)^{2} \\
& =g^{\prime}(u)\left(u_{t}-\delta f(v)\right)+\delta g^{\prime \prime}(u)\left(u_{x}\right)^{2} \\
& \geq g^{\prime}(u) W(x, t)
\end{aligned}
$$


for all $(x, t) \in(0,1) \times\left(\tau_{1}, \tau_{2}\right)$. Since $u_{x}(0, t)=u_{x}(1, t)=0$ and $v_{x}(0, t)=v_{x}(1, t)=0$, we have

$$
W_{x}(0, t)=W_{x}(1, t)=0 \text { and } Z_{x}(0, t)=Z_{x}(1, t)=0, t \in\left[\tau_{1}, \tau_{2}\right] .
$$

By Equations (3) and (4), we have

$$
W(x, 0)=u_{0}^{\prime \prime}(x)-f\left(v_{0}(x)\right)-\delta f\left(v_{0}(x)\right) \text { and } Z(x, 0)=v_{0}^{\prime \prime}(x)-g\left(u_{0}(x)\right)-\delta g\left(u_{0}(x)\right), x \in[0,1] .
$$

If $\delta$ is small enough, then $W(x, 0) \geq 0$ and $Z(x, 0) \geq 0$. By Theorem 2 , we derive that

$$
W(x, t) \geq 0 \text { and } Z(x, t) \geq 0,(x, t) \in[0,1] \times\left[\tau_{1}, \tau_{2}\right] .
$$

Therefore, $u_{t}(x, t) \geq \delta f(v(x, t))$ and $v_{t}(x, t) \geq \delta g(u(x, t))$ in $[0,1] \times(0, T)$.

Next, we prove that the time derivatives blow up when quenching occurs. Blow-up of time derivatives means $u_{t} \rightarrow \infty$ and $v_{t} \rightarrow \infty$ as $t$ approaches the quenching time.

Theorem 5. Let the initial conditions satisfy Equations (5) and (6).

(i) If $v$ quenches in a finite time $T_{q}$, then $u_{t}(1, t)$ blows up at $T_{q}$.

(ii) If $u$ quenches in a finite time $T_{q}$, then $v_{t}(1, t)$ blows up at $T_{q}$.

Proof. We give the proof of (i). One can prove (ii) by using a similar argument. If $v$ quenches in a finite time $T_{q}$, then $v$ quenches only at $x=1$ by Theorem 4 . Thus, $v(1, t) \rightarrow c^{-}$as $t \rightarrow T_{q}^{-}$. By Lemma 4 , we have

$$
u_{t}(1, t) \geq \delta f(v(1, t))
$$

By the hypothesis of $f$, we can conclude that $u_{t}(1, t) \rightarrow \infty$ as $t \rightarrow T_{q}^{-}$.

\section{Simultaneous and Non-Simultaneous Quenching}

In this section, we provide sufficient conditions for simultaneous and non-simultaneous quenching. Moreover, if quenching is non-simultaneous, we give the esimates of the quenching rates.

\section{Theorem 6.}

(i) If $g$ is integrable on $[0, c)$, for any initial condition $v_{0}(x)$, there exists an initial condition $u_{0}(x)$ such that $u$ quenches in a finite time $T_{q}$ while $v$ does not quench at $T_{q}$.

(ii) If $f$ is integrable on $[0, c)$, for any initial condition $u_{0}(x)$, there exists an initial condition $v_{0}(x)$ such that $v$ quenches in a finite time $T_{q}$ while $u$ does not quench at $T_{q}$.

Proof. We only give the proof of (i). One can prove (ii) by using a similar argument. Assume that $g$ is integrable on $[0, c)$. Let $v_{0}(x)$ be fixed. Thus, by Theorem $3, u$ quenches at a finite time $T_{q}$. By Equation (11), we have

$$
\int_{0}^{1}(c-u(x, t)) d x \leq \int_{0}^{1}\left(c-u_{0}(x)\right) d x-t \int_{0}^{1} f\left(v_{0}(x)\right) d x, t \in[0, T]
$$

At the quenching time $T_{q}$, we have

$$
T_{q} \int_{0}^{1} f\left(v_{0}(x)\right) d x \leq c-\int_{0}^{1} u_{0}(x) d x .
$$


For any fixed $\tau \in(0, T)$, let us define $W(x, t)=v_{t}(x, t)$ in $[0,1] \times[0, \tau]$. We have by Equation (2) that

$$
W_{t}(x, t)=\left(u_{t}\right)_{t}(x, t)=u_{x x t}+f^{\prime}(v(x, t)) v_{t}(x, t)=W_{x x}(x, t)+f^{\prime}(v(x, t)) v_{t}(x, t)
$$

for $(x, t) \in(0,1) \times(0, \tau)$. By Lemma 3 (ii), we have $W_{t}(x, t)-W_{x x}(x, t) \geq 0$ for $(x, t) \in(0,1) \times(0, \tau)$. By the boundary conditions of Equation (2), we have

$$
W_{x}(0, t)=0 \text { and } W_{x}(1, t)=0 .
$$

for $t \in(0, T]$. By (4), we have $W(x, 0) \geq f\left(v_{0}(x)\right)$ for $x \in[0,1]$. By Theorem 1 , we obtain

$$
W(x, t) \geq f\left(v_{0}(x)\right),(x, t) \in[0,1] \times[0, T] .
$$

Therefore,

$$
u_{t}(x, t)-f\left(v_{0}(x)\right) \geq 0,(x, t) \in[0,1] \times[0, T] .
$$

Integrating Equation (13) from $t$ to $T_{q}$, we obtain

$$
u(x, t) \leq c-f\left(v_{0}(x)\right)\left(T_{q}-t\right),(x, t) \in[0,1] \times\left[0, T_{q}\right] .
$$

By Equations (2) and (14), we have

$$
v_{t}(x, t) \leq v_{x x}(x, t)+g\left(c-f\left(v_{0}(x)\right)\left(T_{q}-t\right)\right),(x, t) \in[0,1] \times\left[0, T_{q}\right] .
$$

We consider the following problem with the solution $v^{*}(x, t)$,

$$
\left.\begin{array}{ll}
v_{t}^{*}(x, t)=g\left(c-f\left(v_{0}(x)\right)\left(T_{q}-t\right)\right), & t \in(0, T), \\
v^{*}(x, 0)=\max _{x \in[0,1]} v_{0}(x), & x \in[0,1] .
\end{array}\right\}
$$

Integrating the differential equation in Equation (15) from 0 to $t$, we obtain

$$
v(x, t) \leq v^{*}(x, 0)+\int_{0}^{t} g\left(c-f\left(v_{0}(x)\right)\left(T_{q}-\tau\right)\right) d \tau
$$

for $(x, t) \in[0,1] \times\left[0, T_{q}\right]$. From (16), if $u_{0}(x)$ is small enough, we have

$$
v\left(x, T_{q}\right) \leq v^{*}(x, 0)+\lim _{t \rightarrow T_{q}^{-}} \int_{0}^{t} g\left(c-f\left(v_{0}(x)\right)\left(T_{q}-\tau\right)\right) d \tau<c, x \in[0,1] .
$$

Theorem 7. If $f$ and $g$ are not integrable on $[0, c)$, then simultaneous quenching occurs in a finite time.

Proof. We will prove the contrapositive version: "if non-simultaneous quenching occurs in a finite time, then $f$ or $g$ is integrable on $[0, c)$." Assume non-simultaneous quenching occurs in a finite time $T_{q}$ and $u$ is the only solution that quenches at a finite time $T_{q}$. Suppose, for the sake of contradiction, that $g$ is not integrable on $[0, c)$. By Lemma 4 ,

$$
v_{t}(1, t) \geq \delta g(u(1, t)), t \in\left(0, T_{q}\right) .
$$


Integrating Equation (17) from 0 to $t$, we obtain

$$
v(1, t)-v(1,0) \geq \delta \int_{0}^{t} g((u(1, \tau)) d \tau
$$

Then,

$$
\lim _{t \rightarrow T_{q}^{-}} v(1, t)-v(1,0) \geq \delta \lim _{t \rightarrow T_{q}^{-}} \int_{0}^{t} g((u(1, \tau)) d \tau
$$

This is a contradiction; hence, $g$ must be integrable on $[0, c)$. Similary, if we assume non-simultaneous quenching occurs, and $v$ is the only solution that quenches in a finite time, then we have that $f$ is integrable on $[0, c)$.

Theorem 7 implies that, if both $f$ and $g$ are not integrable on $[0, c)$, then quenching is simultaneous for every pair of initial conditions $\left(u_{0}(x), v_{0}(x)\right)$. Next, we impose one more condition to Theorem 6 so that non-simultaneous quenching occurs for every pair of initial conditions $\left(u_{0}(x), v_{0}(x)\right)$. In order to prove the next theorem, let us give the remarks about the estimates of the time derivatives as follows.

At $x=1$, by Lemma 3 (i) and Lemma 4, we have

$$
\left.\begin{array}{l}
\delta f(v(1, t)) \leq u_{t}(1, t) \leq f(v(1, t)) \\
\delta g(u(1, t)) \leq v_{t}(1, t) \leq g(u(1, t)) .
\end{array}\right\} .
$$

Therefore, $\widehat{u}(t)=u(1, t)$ and $\widehat{v}(t)=v(1, t)$ behave as solutions of the system

$$
\left.\begin{array}{l}
\widehat{u}^{\prime}(t)=f(\widehat{v}(t)), t \in(0, T), \\
\widehat{v}^{\prime}(t)=g(\widehat{u}(t)), t \in(0, T) .
\end{array}\right\} .
$$

\section{Theorem 8.}

(i) If $f$ is integrable on $[0, c)$ and $g$ is not integrable on $[0, c)$, then $u(x, t)<c$ for $(x, t) \in[0,1] \times\left[0, T_{q}\right]$.

(ii) If $g$ is integrable on $[0, c)$ and $f$ is not integrable on $[0, c)$, then $v(x, t)<c$ for $(x, t) \in[0,1] \times\left[0, T_{q}\right]$.

Proof. We give the proof of (i). The proof of (ii) can be done by using a similar argument. Suppose $u$ quenches in a finite time $T_{q}$. By Equation (18) and the positivity of $g(\widehat{u}(t))$ for $t \in[0, T]$, we have

$$
\delta g(\widehat{u}(t)) f(\widehat{v}(t)) \leq g(\widehat{u}(t)) \widehat{u}^{\prime}(t) \leq g(\widehat{u}(t)) f(\widehat{v}(t)),
$$

for $t \in(0, T)$. By Equation (19), we have

$$
\delta f(\widehat{v}(t)) \widehat{v}^{\prime}(t) \leq g(\widehat{u}(t)) \widehat{u}^{\prime}(t) \leq f(\widehat{v}(t)) \widehat{v}^{\prime}(t),
$$

for $t \in(0, T)$. Integrating Equation (20) from 0 to $t$, we obtain

$$
\int_{0}^{t} g(\widehat{u}(\tau)) d \widehat{u}(\tau)=\int_{0}^{t} g(\widehat{u}(\tau)) \widehat{u}^{\prime}(\tau) d \tau \leq \delta \int_{0}^{t} f(\widehat{v}(\tau)) \widehat{v}^{\prime}(\tau) d \tau=\delta \int_{0}^{t} f(\widehat{v}(\tau)) d \widehat{v}(\tau) .
$$

As $t$ approaches the quenching time $T_{q}$, we have

$$
\lim _{t \rightarrow T_{q}^{-}} \int_{0}^{t} g(\widehat{u}(\tau)) d \widehat{u}(\tau) \leq \delta \lim _{t \rightarrow T_{q}^{-}} \int_{0}^{t} f(\widehat{v}(\tau)) d \widehat{v}(\tau)
$$

Since $u$ quenches in a finite time $T_{q}$, this is a contradiction. The theorem is proved.

\section{Theorem 9.}


(i) If quenching is non-simultaneous and $u$ is the only solution that quenches at $T_{q}$, then

$$
c-\int_{t}^{T_{q}} f(v(1, \tau)) d \tau \leq u(1, t) \leq c-\delta f\left(v_{0}(x)\right)\left(T_{q}-t\right) .
$$

(ii) If quenching is non-simultaneous and $v$ is the only solution that quenches at $T_{q}$, then

$$
c-\int_{t}^{T_{q}} g(u(1, \tau)) d \tau \leq v(1, t) \leq c-\delta g\left(u_{0}(x)\right)\left(T_{q}-t\right) .
$$

Proof. We give the proof of (i). The proof of (ii) can be done by using a similar argument. Assume non-simultaneous quenching occurs and $u$ is the only solution that quenches in a finite time $T_{q}$. Hence, $u$ quenches only at $x=1$. By Lemma 4 ,

$$
u_{t}(1, t) \geq \delta f(v(1, t)),
$$

for $t \in(0, T)$. By Lemma 3 and $f$ being an increasing function, we have

$$
u_{t}(1, t) \geq \delta f\left(v_{0}(x)\right)
$$

for $t \in(0, T)$. Integrating Equation (21) with respect to $t$ from $t$ to $T_{q}$, we obtain

$$
u\left(1, T_{q}\right)-u(1, t) \geq \delta f\left(v_{0}(x)\right)\left(T_{q}-t\right) .
$$

Since $u(1, t) \rightarrow c^{-}$as $t \rightarrow T_{q}^{-}$, this gives the upper estimate of $u(1, t)$ as

$$
u(1, t) \leq c-\delta f\left(v_{0}(x)\right)\left(T_{q}-t\right)
$$

Next, we find the lower estimate of $u(1, t)$. From the System (2) and (18), we have

$$
u_{t}(1, t) \leq f(v(1, t))
$$

for $t \in(0, T)$. Integrating Equation (23) with respect to $t$ from $t$ to $T_{q}$, we obtain

$$
u\left(1, T_{q}\right)-u(1, t) \leq \int_{t}^{T_{q}} f(v(1, \tau)) d \tau
$$

Since $u(1, t) \rightarrow c^{-}$as $t \rightarrow T_{q}^{-}$, we have the lower estimate of $u(1, t)$ as

$$
u\left(1, T_{q}\right)-\int_{t}^{T_{q}} f(v(1, \tau)) d \tau \leq u(1, t) .
$$

Therefore, combing of Equations (22) and (24), we have the quenching rate of $u(1, t)$ as $t$ approaches $T_{q}$.

Theorem 9 implies that, if non-simultaneous quenching occurs, then the rate at which the quenched solution approaches zero is of linear order.

\section{Discussion and Conclusions}

We studied the system of coupled semi-linear heat equations. The existence result is proved by using comparison principles and the technique of upper and lower solutions. Under proper conditions, we prove that the finite time quenching occurs to the system, and we also provide the queching set. We obtain the sufficient conditions for simultaneous and non-simultaneous quenching. The blow-up of time derivatives of the solutions at the queching point are proved. Moreover, we observe that the integrability of $f$ or $g$ plays an important role to prevent one of the solutions from 
quenching in a finite time. For non-simultaneous quenching, we give the estimates of the quenching rates. Finally, we propose that our future research is to extend our study to the $n$-dimensional domain.

Author Contributions: Conceptualization, R.B., T.T., and A.-a.W.; methodology, R.B., T.T., and A.-a.W.; validation, R.B., T.T., and A.-a.W.; formal analysis, R.B., T.T., and A.-a.W.; investigation, R.B., T.T., and A.-a.W.; writing-original draft preparation, A.-a.W.; writing—review and editing, R.B. and T.T.; supervision, R.B. and T.T.; project administration, R.B. and T.T. All authors have read and agreed to the published version of the manuscript.

Funding: This research received no external funding.

Conflicts of Interest: The authors declare no conflict of interest.

\section{References}

1. Kawarada, H. On solutions of initial boundary problem for $u_{t}=u_{x x}+\frac{1}{1-u}$. Publ. RIMS Kyoto Univ. 1975, 10, 729-736. [CrossRef]

2. De Pablo, A.; Quirós, F.; Rossi, J.D. Nonsimultaneous quenching. Appl. Math. Lett. 2002, 15, $265-269$. [CrossRef]

3. Pei, H.; Li, Z. Quenching for a parabolic system with general singular terms. J. Nonlinear Sci. Appl. 2016, 9, 5281-5290. [CrossRef]

4. Chan, W.Y. Simultaneous quenching for semilinear parabolic system with localized sources in a square domain. J. Appl. Math. Phys. 2019, 7, 1473-1487. [CrossRef]

5. Fornaro, S. Regularity Properties for Second Order Partial Differential Operators with Unbounded Coefficients. Ph.D. Thesis, Department of Mathematics and Physics Ennio De Giorgi, University of Salento, Lecce, Italy, 2004.

6. Friedman, A. Partial Differential Equations of Parabolic Type; Prentice Hall: Upper Saddle River, NJ, USA, 1964.

7. Protter, M.H.; Weinberger, H.F. Maximum Principles in Differential Equations; Progress in USA; Springer: Hoboken, NJ, USA, 1984.

8. Fu, S.C.; Guo, J.S. Blow-up for a semilinear reaction-diffusion system coupled in both equations and boundary conditions. J. Math. Anal. Appl. 2002, 276, 458-475. [CrossRef]

9. Selçuk, B. Quenching behavior of a semilinear reaction-diffusion system with singular boundary condition. Turk. J. Math. 2016, 40, 166-180. [CrossRef]

Publisher's Note: MDPI stays neutral with regard to jurisdictional claims in published maps and institutional affiliations.

(C) 2020 by the authors. Licensee MDPI, Basel, Switzerland. This article is an open access article distributed under the terms and conditions of the Creative Commons Attribution (CC BY) license (http://creativecommons.org/licenses/by/4.0/). 